\title{
BMJ Open Potentially avoidable and ambulatory care sensitive hospitalisations among forced migrants: a protocol for a systematic review and meta-analysis
}

\author{
Célina Lichtl, Sandra Claudia Gewalt, Stefan Noest, Joachim Szecsenyi, \\ Kayvan Bozorgmehr
}

To cite: Lichtl C, Gewalt SC, Noest $\mathrm{S}$, et al. Potentially avoidable and ambulatory care sensitive hospitalisations among forced migrants: a protocol for a systematic review and meta-analysis. BMJ Open 2016;6:e012216. doi:10.1136/bmjopen-2016012216

- Prepublication history and additional material is available. To view please visit the journal (http://dx.doi.org/ 10.1136/bmjopen-2016012216).

Received 11 April 2016 Revised 8 June 2016 Accepted 1 August 2016

CrossMark

Department of General Practice \& Health Services Research, University Hospital Heidelberg, Heidelberg, Germany

\section{Correspondence to} Dr Kayvan Bozorgmehr; kayvan.bozorgmehr@med. uni-heidelberg.de

\section{ABSTRACT}

Introduction: There is an increasing number of forced migrants globally, including refugees, asylum seekers, internally displaced persons and undocumented migrants. According to international law, forced migrants should enjoy access to health services free of discrimination equivalent to the host population, but they face barriers to healthcare worldwide. This may lead to a delay in care and result in preventable hospital treatment, referred to as potentially preventable hospitalisation (PPH) or ambulatory care sensitive hospitalisation (ACSH). There is as yet no overview of the prevalence of PPH in different countries and groups of forced migrants, and it is unknown whether the concept has been used among these migrant groups. We aim to systematically review the evidence (1) on the prevalence of PPH among forced migrants and (2) on differences in the prevalence of PPH between forced migrants and the general host population.

Methods and analysis: A systematic review will be conducted searching databases (PubMed/MEDLINE, Web of Science/Knowledge, Cochrane Library, CINAHL, Google Scholar) and the internet (Google). Inclusion criteria: observational studies on forced migrants reporting PPH or ACSH with or without comparison groups published in the English or German language. Exclusion criteria: studies on general migrant groups or hospitalisations without clear reference to avoidability. Study selection: titles, abstracts and full texts will be screened in duplicate for eligibility. Data on the prevalence of PPH/ACSH among forced migrants, as well as any reported prevalence differences between host populations, will be systematically extracted. Quality appraisal will be performed using standardised checklists. The evidence will be synthesised in tabular form and by means of forest plots. A meta-analysis will be performed only among homogeneous studies (in terms of design and population).

Ethics and dissemination: Ethical clearance is not necessary (secondary research). The results will be disseminated via publication in open access journals, conferences and public media.

PROSPERO registration number: CRD42016037081.
Strengths and limitations of this study

- The first systematic review and meta-analysis on observational studies on preventable hospitalisations and ambulatory care sensitive conditions among forced migrants.

- Will provide highest evidence on access to timely and effective primary care for forced migrants.

- A broad search in five databases and consideration of the grey literature will show if and how the concepts of preventable hospitalisations and ambulatory care sensitive conditions have been applied among the heterogeneous population of forced migrants.

- The analysis on differences in the outcome of interest between forced migrants and the general host population will be strongly dependent on the existence of comparative studies.

- Comparability of findings may be limited due to the heterogeneity of both the group of forced migrants and the policy contexts at country level.

\section{BACKGROUND}

Millions of people have been forced globally from their homes by civil conflicts, regional wars and political violence. The latest figures from the United Nations High Commissioner for Refugees (UNHCR) are alarming: in 2014, forced displacement has demonstrated an accelerated growth reaching unparalleled numbers with the highest recorded number of people being displaced since records began. At the end of 2014, 59.5 million people were forcibly displaced globally, accounting for an increase of 8 million individuals compared with $2013 .{ }^{1}$ More than half $(53 \%)$ of all refugees came from three countries: the Syrian Arab Republic (3.88 million), Afghanistan (2.59 million) and Somalia ( 1.11 million). ${ }^{1}$ The largest refugeehosting countries worldwide were Turkey, Pakistan, Lebanon and the Islamic Republic 
of Iran. ${ }^{1}$ In $2014,51 \%$ of the 59.5 million refugees were under the age of $18 .{ }^{1}$

A large proportion of refugees are internally displaced persons, who have searched for shelter and safety within the international borders of their home country. The Internal Displacement Monitoring Centre (IDMC) highlighted that internal displacement induced by conflict and violence affected over 38 million people at the end of 2014, which amounts to around $64 \%$ of forcibly displaced persons worldwide. ${ }^{2}$ Disaster-induced displacement accounted for an average of 26 million people per year since 2008, an equivalent of one person being displaced every second. ${ }^{3}$

A smaller but still significant number is that of asylum seekers, whose claim for refugee status has not yet been definitively evaluated. They accounted for around 1.8 million people in $2014 .{ }^{1}$ However, owing to failed recognition as refugees, visa overstay or irregular entry into respective countries, there is a considerable number of irregular or undocumented migrants, with trends expected to grow. Data on flows and trends of irregular migration vary widely and are usually imprecise, especially on a global level. The overall estimate for the 27 European Union Member States ranged from 1.9 to 3.8 million undocumented migrants in $2008 .{ }^{4}$

These different groups of migrants can be referred to as forced mixed migrant flows, a heterogeneous group comprising refugees, internally displaced persons, asylum seekers and undocumented migrants.

The 1951 Refugee Convention states that refugees should enjoy access to health services equivalent to that of the host population. ${ }^{5}$ Under international law, everybody has the right to the highest standards of physical and mental health and this includes the right to healthcare free of discrimination, according to article 12 of the International Covenant of Economic, Social and Cultural Rights. ${ }^{6}$ Access to healthcare is, however, mostly limited or actively restricted for refugees and other forcibly displaced migrants. ${ }^{7-10}$ Barriers to healthcare-which are shaped by inequalities in availability, access and quality of services, by the financial burden these may impose on people, and even by linguistic, cultural and gender-based factors ${ }^{11}$ - can lead to a delay in seeking and receiving care, which in return can result in costly-and possibly preventable-hospital treatment. ${ }^{12}$

Regarding the many possible stages ${ }^{13}$ throughout the migration process, in which (forced) migrants can be exposed to health risks, there is a broad range of potential health conditions migrants may have to deal with. Influences on health in the 'predeparture' stage of the migration process could be local chronic disease patterns and pathogens, local cultures and lifestyle, as well as environmental factors. Political or personal circumstances, such as human rights violations or interpersonal violence, may affect the psychological and physical health status throughout the journey, particularly in the case of forced migrants. ${ }^{13}$ During the perimigration phase, health influences are closely related to the mode of transport and circumstances of travel. Furthermore, in this phase, pathogens may be acquired or carried across different zones of disease prevalence. Once migrants settle down in their intended location, irrespective of the duration, attention may be required for non-communicable diseases, mental health and socioeconomic influences on health. ${ }^{13}$ Forced migrants also often experience situations of temporary detention or interim residence: immigration detention centres or refugee camps may have deleterious effects on mental or physical health and may be unhygienic or unsafe. ${ }^{13}$

The concept of potentially preventable hospitalisations (PPH) or its specific subcategory ambulatory care sensitive hospitalisations (ACSH) have been used as population-based indicators to assess the quality and strength of primary care. ${ }^{14}$ The concept of ACSH is based on the assumption that hospitalisation rates can be reduced by effective ambulatory care, whereas $\mathrm{PPH}$ use a much broader approach and include a spectrum of population-based interventions and social measures. ${ }^{15}$ Accordingly, sets of ambulatory care sensitive conditions (ACSCs) often include conditions for which acute management should prevent admission, for example, dehydration and gastroenteritis, and chronic conditions where preventative care should prevent later admission, for example, complications of diabetes. ${ }^{16}$ It can also include infectious diseases, which could have been managed by timely and effective immunisation. The relationship between the quality of ambulatory care and ACSH is influenced by various exogenous factors, ${ }^{17}$ including patient demographics, ${ }^{18-21}$ disease burden, ${ }^{21-23}$ behavioural risk ${ }^{22}$ socioeconomic factors, ${ }^{18}{ }^{21}{ }^{23-29}$ the structure of the hospital sector, ${ }^{24}{ }^{30}$ patient preferences regarding use of care ${ }^{22}$ and compliance. ${ }^{31}$ The structure of the healthcare system ${ }^{32}$ (and thus the role of primary and hospital care) may also be of importance in the concept of ACSCs and ACSH. Recent systematic reviews confirm the validity of using hospitalisations for ACSCs as an indicator of primary care quality conditional on the application of appropriate adjustment factors. ${ }^{32}$

The concept of PPH with its specific category of ACSH could thus be a useful indicator to assess the quality of primary care accessible to forced migrants or specific vulnerable subgroups (such as children, pregnant women or people with disabilities) in different settings and countries. It could also be used to assess disparities in access between forced migrants and the host population. There is, however, no overview of the prevalence of $\mathrm{PPH}$ or ACSH in different countries or groups of forced migrants, and it is unknown whether the concept has been used at all among these migrant groups. An initial search in the Cochrane Library of Systematic Reviews and a prospective register of systematic reviews (PROSPERO) using the terms asylum* or refugee* linked with terms for ACSH identified no reviews.

The objectives of this study are thus to systematically review: 
1. The evidence on the prevalence of PPH among forced migrants and specific vulnerable subgroups (children, pregnant women, people with disabilities).

2. The evidence for differences in the prevalence of PPH between forced migrants and the general host population.

\section{METHODS AND DESIGN}

Study design

We will conduct a systematic review according to the 'Cochrane Handbook for Systematic Reviews of Interventions' ${ }^{33}$ The guidelines for the inclusion of non-randomised studies will be taken into special consideration. $^{34}$

\section{Review questions}

We used the PICO criteria (population, intervention, comparison and outcome) to formulate the questions to be addressed by the systematic review:

1. What is the prevalence of PPH among forced migrants and specific vulnerable subgroups (children, pregnant women, people with disabilities)?

2. Are there differences in the prevalence of $\mathrm{PPH}$ between forced migrants and the general host population?

\section{Search strategy for identification of studies}

The search strategy includes searching databases for relevant articles, which match our predefined inclusion criteria (see below) as well as searching the internet for grey literature.

\section{Search strategy 1: databases}

We designed the search strategy according to the 'Cochrane Handbook for Systematic Reviews of Interventions' ${ }^{33}$ The following electronic bibliographic databases will be searched for studies:

- PubMed/MEDLINE;

- Web of Science Core Collection, including:

- Social Sciences Citation Index (SSCI);

- Science Citation Index Expanded (SCI-EXP).

- Cochrane Library (without limitation to specific databases);

- CINAHL.

The search engine 'Google Scholar' will be used to search further academic databases.

\section{Search strategy 2: internet search}

To identify grey literature, an internet search will be carried out using the search engine Google. This search may present further relevant studies not published in conventional academic journals.

\section{Search terms}

The search terms (table 1) were developed according to PICO criteria. Medical Subject Headings (MeSH) terms were used to identify synonyms for the population and
Table 1 Search terms for the systematic review

\begin{tabular}{|c|c|c|c|}
\hline Population & Intervention & Comparison & Outcome \\
\hline $\begin{array}{l}\text { asylum } \\
\text { seeker*; }^{*} \text { asyl*; }^{*} \\
\text { refugee }^{*} ; \\
\text { displaced; } \\
\text { migra* }^{*} \\
\text { forced migra*; } \\
\text { undocumented; } \\
\text { sans papier; } \\
\text { irregular; } \\
\text { illegal; } \\
\text { unauthorized }\end{array}$ & - & $\begin{array}{l}\text { general } \\
\text { population }\end{array}$ & $\begin{array}{l}\text { ambulatory } \\
\text { care } \\
\text { ambulatory } \\
\text { care } \\
\text { sensitive- } \\
\text { preventable- } \\
\text { avoidable- } \\
\text { hospitali* }^{*} \\
\text { admission* } \\
\text { condition }\end{array}$ \\
\hline
\end{tabular}

the outcome. Terms for the comparison (the general host population) were not included in the search combination to allow for the inclusion of descriptive epidemiological studies without comparison groups. A librarian based at the University Heidelberg was consulted to develop the Boolean search combination.

The search terms within columns will be linked by the operator 'OR' and terms between columns by the operator 'AND' to search for potentially relevant articles in titles, abstracts and full texts. The aforementioned databases and search engines will thus be searched without restrictions using the following search term combination:

(asylum seeker* OR asyl* OR refugee* OR forced migra* OR migra* OR displaced OR undocumented OR sans papier* OR unauthorized OR illegal* OR irregular) AND ('ambulatory care' OR 'ambulatory care sensitive' OR avoidable OR preventable) AND (hospitali* OR admission* OR condition).

The internet search will be restricted to the English language and PDF documents. An overview of the exact search terms used by the database/search engine is provided in online supplementary file 1 .

\section{Data management}

Records will be downloaded and stored in a reference management software (EndNote). After exclusion of duplicates, an excel file will be created to perform the screening process.

\section{Selection of studies-eligibility criteria} Inclusion criteria

Studies will be considered as eligible for inclusion if they fulfill the following criteria:

- Population: Refugees, (forcibly) displaced persons, asylum seekers and sans papiers, undocumented, illegal, irregular or unauthorised migrants as the study population. We will only include studies on 'migrants' if there is a clear distinction between forced migrants (refugees, asylum seekers, displaced persons and sans papiers, undocumented, illegal, 
irregular or unauthorised migrants) and regular forms of migration.

- Study design: Quantitative observational studies, that is, cohort and case-control studies, cross-sectional studies, and descriptive surveys using primary data or routine/secondary data on $\mathrm{PPH}$.

- Types of articles: Original articles/reports of primary research; systematic and non-systematic reviews of observational studies.

- Outcome measure. Studies on PPH or related concepts as reported by primary studies (eg, avoidable hospitalisations, ACSH or hospitalisations avoidable through prevention).

- Geographical area: All articles irrespective of their geographical area or geographical focus will be included.

- Language: Articles published in German or English.

- Date of publication: No restrictions on the date of publication.

\section{Exclusion criteria}

- Population: Migrants without clear reference to forced migration (asylum seeker status/refugee status, internally displaced persons and sans papiers, undocumented, illegal, irregular or unauthorised migrants).

- Study design: Qualitative studies.

- Types of articles: Newspaper articles, expert opinions, commentaries, discussion papers, journalistic interviews, policy reports, books, conference proceedings, abstracts.

- Outcome measure. Studies reporting hospitalisations without a clear link to preventability of these hospitalisations or sensitivity to ambulatory care.

- Language. Articles not published in German or English.

- Accessibility: Articles that are not available in full text.

\section{Screening process}

The screening process will consist of two steps:

1. Title and abstract screening;

2. Full-text screening.

\section{Title and abstract screening}

After removal of duplicates, two reviewers will independently screen $10 \%$ of the articles by title and abstract, and decide on inclusion or exclusion of the articles based on the previously defined criteria. If the screening process of the initial $10 \%$ of articles indicates the necessity to perform alterations in inclusion and exclusion criteria, redefinitions will be executed at this stage. In the next step, two reviewers will independently screen all article titles and abstracts taking the (potentially redefined) inclusion/exclusion criteria into consideration. Articles that prove to be suitable for inclusion from the initial and subsequent screening processes will be recorded in an Excel File/EndNote database. If the two reviewers disagree on the eligibility of an article, the whole review team will discuss it until a consensus is achieved. In the case of discrepancies in judgement on eligibility in the absence of clear exclusion criteria, all publications that are considered to be relevant by at least one reviewer will be obtained as full text and assessed for eligibility in the next stage.

\section{Full-text screening}

After screening titles and abstracts, two reviewers will independently screen the full text of the previously selected articles to assess their eligibility for inclusion in the final review. Discrepancies in judgements between the two reviewers will be discussed within the review team. Only references that are considered to be eligible by all members of the review team will be included.

\section{Data extraction and critical appraisal}

Data extraction and critical appraisal will be performed after full-text screening.

\section{Data extraction}

On the basis of the STROBE ${ }^{35}$ checklist and information relevant to the objective of the study, a preliminary data extraction form (see online supplementary file 2) has been designed to systematically extract the following pieces of information:

- Generic bibliographic information (author, year published);

- Study characteristics (year of study/study period/ research method);

- Study objectives/research questions;

- Population (age, sex, migrant status, country of origin, vulnerability, ie, affiliation to subgroups such as children, pregnant women, people with disabilities);

- Context characteristics (study setting, country in which study is performed);

- Form of hospitalisation described: potentially avoidable/preventable or ACSH;

- Measures of frequency/association for the analysed outcomes including SEs and/or $95 \%$ CIs;

- For analytical studies: exposures and covariables and cofounders on individual and/or contextual level;

- Main results and limitations of the study as reported;

- Results of the critical appraisal.

If needed, the data extraction form will be adapted and re-evaluated as the research proceeds. Two reviewers will perform data extraction in duplicate independently. All articles will be checked vice versa by the senior author of the protocol. Authors of primary studies will be contacted if necessary to obtain or confirm relevant data.

\section{Critical appraisal}

Case-control and cohort studies will be evaluated by means of the Newcastle-Ottawa Scale for nonrandomised studies (NOS) ${ }^{36}$ Cross-sectional studies will be assessed using the tool for 'Critical Appraisal of Cross-sectional Studies' by the National Collaborating 
Centre for Environmental Health. ${ }^{37}$ The AMSTAR tool, a validated 11-item tool to assess the quality of systematic reviews of intervention studies, will appraise the quality of reviews. ${ }^{38}$ This tool will also be used to assess nonsystematic reviews in order to evaluate their quality against the 'gold standard' of systematic reviews. Non-applicable items will not be weighted in order to avoid inappropriate judgements on the quality caused by the (potential) non-applicability of AMSTAR items.

The aim of the critical appraisal process will be to evaluate the risk of bias in reported results, not to exclude literature.

\section{Analysis and evidence synthesis}

Tables will be created to give an overview on the data that have been extracted from the data extraction forms (ie, a description of included studies, study populations, methods, results and quality). We will draw one or more forest plots of the extracted data on the prevalence of PPH and/or respective measures of association from comparative studies using reference groups from the general population. If sufficient detail is provided in primary reports, the results will be presented stratified by sex and/or special subgroups such as children, pregnant women or people with disabilities. A meta-analysis according to the guidelines for meta-analysis of observational studies in epidemiology (MOOSE) guidelines ${ }^{39}$ will be performed only among studies which are homogeneous with respect to both study design and population. To assess inconsistencies across studies and their impact on pooled estimates, the percentage of the variability in effect estimates that is due to statistical heterogeneity rather than chance $\left(\mathrm{I}^{2}\right)$ will be calculated. Random-effects meta-analysis will be performed in case of substantial heterogeneity $\left(\mathrm{I}^{2}>50 \%\right)$ that cannot be explained among studies that would otherwise be considered suitable for a meta-analysis. Otherwise, fixed-effects meta-analysis will be performed.

\section{DISCUSSION}

This systematic review will provide an overview and synthesis of research on the prevalence of PPH and ACSH among forced migrant populations. Given the challenges of refugee flows and later refugee resettlement, it is important for governments, public health professionals and health service providers in resettlement countries to obtain evidence on humanitarian arrivals' access to primary care and preventable hospitalisations. ${ }^{40}$

The outcomes of PPH have been known to be difficult to assess and we would like to assemble the information on the concept itself and further explore the extent of its application in studies, thus evaluating if it has been established as a useful measurement tool in healthcare research among forced migrants.

The results could serve as a foundation for future research on this topic in order to provide evidence for internationally comparative research or for interventions to reduce PPH among forced migrants. The results of our review are a cornerstone in evidence related to $\mathrm{PPH}$ in this specific population. Hence, they can be used to identify potential targets related to avoidable hospitalisations and areas for interventions, aimed at improving both health status and appropriate access to healthcare among forced migrants through sufficient access to primary care, population-based interventions and preventive measures.

The strength of this review is the inclusion of observational studies, the clearly defined inclusion and exclusion criteria, the transparent and systematic search strategy, and its approaches for screening, extracting and assessing the available research. It also follows clear steps for the analysis of search results by evidence mapping and narrative description of the findings.

Owing to our broad search in five databases and the inclusion of grey literature, we will have an answer to the overall question if and how the concepts of preventable hospitalisations and ambulatory care sensitive conditions among the heterogeneous population of forced migrants have been applied. The analysis on differences in the outcome of interest between forced migrants and the general host population will be strongly dependent on the existence of comparative studies. We will have to consider that comparability of findings may be limited due to the heterogeneity of both the group of forced migrants and the policy contexts at country level.

With this review, we aim at providing essential information for health professionals and policymakers to improve understanding of persisting barriers and potential benefits of improved access (ie, availability, geographic accessibility, social acceptability and financial affordability) to primary care for forced migrants and to increase evidence-based decision-making. We hope that the information gained on PPH among forced migrants will be of use to policymakers when evaluating their approaches on access to healthcare for forced migrants.

Targeting both a lively discussion of this topic and a wide dissemination of our findings to different levels of the healthcare system, including policymakers, healthcare providers and researchers, we will publish this review in an open access journal and circulate the results via conferences, civil society organisations and academic institutions.

Acknowledgements The authors thank Mr Dietmar Fleischer (University Library Heidelberg) for his support in developing the search terms for the review.

Contributors KB conceived the study. CL, SCG and KB planned and designed the protocol. CL and SCG wrote the first draft. CL, SCG and KB wrote the subsequent drafts. SN and JS contributed in the critical review and provision of important intellectual content. All the authors have approved and contributed to the final written manuscript.

Funding The study received financial support from the Deutsche Forschungsgemeinschaft and Ruprecht-Karls-Universität Heidelberg within the funding programme Open Access Publishing.

Competing interests None declared.

Provenance and peer review Not commissioned; externally peer reviewed. 
Data sharing statement The results will be disseminated via publication in open access journals, conferences and the public media.

Open Access This is an Open Access article distributed in accordance with the Creative Commons Attribution Non Commercial (CC BY-NC 4.0) license, which permits others to distribute, remix, adapt, build upon this work noncommercially, and license their derivative works on different terms, provided the original work is properly cited and the use is non-commercial. See: http:// creativecommons.org/licenses/by-nc/4.0/

\section{REFERENCES}

1. The UN Refugee Agency, UNHCR Global Trends-Forced Displacement in 2014. 2014, The office of the United Nations High Commissioner for Refugees (UNHCR) 2001-2016.

2. Bilak A, Caterina M, Charron G, et al. General overview 2015people internally displaced by conflict and violence. Lennard J, ed. 2015.

3. Yonetani M, et al. Global estimates 2015-people displaced by disasters. Internal Displacement Monitoring Centre, Norwegian Refugee Council, 2015

4. Vogel D. Size and development of irregular migration to the EU. CLANDESTINO Research Project. Counting the uncountable: data and trends across Europe. European Commission, 2009.

5. General Assembly of the United Nations. Convention relating to the status of refugees. United Nations, ed. Geneva: UNHCR-The UN Refugee Agency, 1951.

6. Committee on Economic, Social and Cultural Rights (CESCR). Substantive issues arising in the implementation of the International Covenant on Economic, Social and Cultural Rights: General Comment No.14 (2000) - the right to the highest attainable standard of health (article 12 of the International Covenant on Economic, Social and Cultural Rights), United Nations, Editor. 2000, Office of the United Nations High Commissioner for Human Rights. 2000.

7. Norredam M, Mygind A, Krasnik A. Access to health care for asylum seekers in the European Union-a comparative study of country policies. Eur J Public Health 2006;16:286-90.

8. Cuadra CB. Right of access to health care for undocumented migrants in EU: a comparative study of national policies. Eur J Public Health 2012;22:267-71.

9. De Vito E, de Waure C, Specchia ML, et al. Public health aspects of migrant health: a review of the evidence on health status for undocumented migrants in the European Region. Copenhagen: World Health Organization, 2015.

10. Bradby H, Humphris R, Newall D, et al. Public health aspects of migrant health: a review of the evidence on health status for refugees and asylum seekers in the European Region. Copenhagen: World Health Organization, 2015.

11. Dans $A M$, Dans $L$, Oxman $A D$, et al. Assessing equity in clinical practice guidelines. J Clin Epidemiol 2007;60:540-6.

12. WHO. The World Health Report 2008-primary health care (Now More Than Ever). Geneva: World Health Organizaiton, 2008.

13. Zimmerman C, Kiss L, Hossain M. Migration and health: a framework for 21st century policy-making. PLoS Med 2011;8:e1001034.

14. Burgdorf $F$, Sundmacher $L$. Potentially avoidable hospital admissions in Germany: an analysis of factors influencing rates of ambulatory care sensitive hospitalizations. Dtsch Arztebl Int 2014;111:215-23.

15. Jackson G, Tobias M. Potentially avoidable hospitalisations in New Zealand, 1989-98. Aust N Z J Public Health 2001;25:212-21.

16. Purdy S, Griffin T, Salisbury $C$, et al. Ambulatory care sensitive conditions: terminology and disease coding need to be more specific to aid policy makers and clinicians. Public Health 2009;123:169-73.

17. Sundmacher L, Fischbach D, Schuettig W, et al. Which hospitalisations are ambulatory care-sensitive, to what degree, and how could the rates be reduced? Results of a group consensus study in Germany. Health Policy 2015;119:1415-23.

18. Barnett $R$, Malcolm $L$. Practice and ethnic variations in avoidable hospital admission rates in Christchurch, New Zealand. Health Place 2010;16:199-208.

19. Longman JM, I Rolfe M, Passey MD, et al. Frequent hospital admission of older people with chronic disease: a cross-sectional survey with telephone follow-up and data linkage. BMC Health Serv Res 2012:12:373.

20. Balogh R, Brownell M, Ouellette-Kuntz $\mathrm{H}$, et al. Hospitalisation rates for ambulatory care sensitive conditions for persons with and withou an intellectual disability - a population perspective. J Intellect Disabil Res 2010;54:820-32.

21. Walker RL, Chen G, McAlister FA, et al. Relationship between primary care physician visits and hospital/emergency use for uncomplicated hypertension, an ambulatory care-sensitive condition. Can J Cardiol 2014;30:1640-8.

22. Ansari Z, Laditka JN, Laditka SB. Access to health care and hospitalization for ambulatory care sensitive conditions. Med Care Res Rev 2006;63:719-41.

23. Giuffrida A, Gravelle H, Roland M. Measuring quality of care with routine data: avoiding confusion between performance indicators and health outcomes. BMJ 1999;319:94-8.

24. Sundmacher L, Kopetsch T. The impact of office-based care on hospitalizations for ambulatory care sensitive conditions. Eur J Health Econ 2015;16:365-75.

25. Trachtenberg AJ, Dik N, Chateau D, et al. Inequities in ambulatory care and the relationship between socioeconomic status and respiratory hospitalizations: a population-based study of a Canadian city. Ann Fam Med 2014;12:402-7.

26. White BM, Ellis CJr, Simpson KN. Preventable hospital admissions among the homeless in California: a retrospective analysis of care for ambulatory care sensitive conditions. BMC Health Serv Res 2014;14:511.

27. Billings J, Zeitel L, Lukomnik J, et al. Impact of socioeconomic status on hospital use in New York City. Health Aff (Millwood) 1993;12:162-73.

28. Blustein J, Hanson K, Shea S. Preventable hospitalizations and socioeconomic status. Health Aff (Millwood) 1998;17:177-89.

29. Billings J, Anderson GM, Newman LS. Recent findings on preventable hospitalizations. Health Aff (Millwood) 1996;15: 239-49.

30. Berlin C, Busato A, Rosemann T, et al. Avoidable hospitalizations in Switzerland: a small area analysis on regional variation, density of physicians, hospital supply and rurality. BMC Health Serv Res 2014;14:289.

31. Freund T, Campbell SM, Geissler S, et al. Strategies for reducing potentially avoidable hospitalizations for ambulatory care-sensitive conditions. Ann Fam Med 2013;11:363-70.

32. Rosano A, Loha CA, Falvo R, et al. The relationship between avoidable hospitalization and accessibility to primary care: a systematic review. Eur J Public Health 2013;23:356-60.

33. Higgins J, Green S. Cochrane handbook for systematic reviews of interventions. Oxford: Cochrane Collaboration, 2011.

34. Reeves B, Deeks JJ, Higgins JP, et al. Chapter 13: Including non-randomized studies. In: Higgins J, Green S. eds. Cochrane Handbook for Systematic Reviews of Interventions. Cochrane Collaboration, 2008:23.

35. Institute of Social and Preventative Medicine (ISPM). STROBE Statement: STROBE checklists. 2007 (cited 21.02.2016). http:// strobe-statement.org/index.php?id=available-checklists

36. Wells GA, Shea B, O'connell D, et al. The Newcastle-Ottawa Scale (NOS) for assessing the quality of nonrandomised studies in meta-analyses. Ottawa Hospital Research Institute, University of Ottawa. 2014

37. National Collaborating Centre for Environmental Health. A primer for evaluating the quality of studies: critical appraisal of cross-sectional studies. Vancouver: National Collaborating Centre for Environmental Health, 2011.

38. Shea BJ, Hamel C, Wells GA, et al. AMSTAR is a reliable and valid measurement tool to assess the methodological quality of systematic reviews. J Clin Epidemiol 2009;62(10):1013-20.

39. Stroup DF, Berlin JA, Morton SC, et al. Meta-analysis of observational studies in epidemiology: a proposal for reporting. JAMA 2000;283:2008-12.

40. Correa-Velez I, Ansari Z, Sundararajan V, et al. A six-year descriptive analysis of hospitalisations for ambulatory care sensitive conditions among people born in refugee-source countries. Popul Health Metr 2007;5:9-9. 